Artigo Revisão

\title{
Freire e formação para o Sistema Único de Saúde: o enfermeiro, o médico e o odontólogo
}

\author{
Freire's theoretical framework and the professional capabilities of nurses, physicians, and dentists \\ for the Brazilian Universal Healthcare System
}

Freire y la formación para el Sistema Único de Salud: el enfermero, el médico y el odontólogo

Rodrigo Otávio Moretti-Pires ${ }^{1}$, Sonia Maria Villela Bueno ${ }^{2}$

\section{RESUMO}

A criação e implementação do Sistema Único de Saúde (SUS) trouxe vitórias como a universalidade, equidade e integralidade na assistência como um dever do Estado. Diversas estratégias foram concebidas, inclusive o Programa de Saúde da Família que em teoria re-significaria a atenção primária no âmbito do SUS. No entanto, deficiências na formação universitária do enfermeiro, do médico e do odontólogo são constatadas pela dificuldade destes profissionais no redirecionamento das ações enfocando a família e promoção da saúde, dentro da necessidade de atuação integral. Neste ínterim, a temática da humanização do atendimento torna-se um emblema privilegiado do paradoxo. Os autores debatem estas questões através do uso de revisão narrativa, promovendo reflexão sobre o marco teórico freireano como caminho na formação universitária para superação destes dilemas na consecução do SUS de acordo com seus ideais e princípios.

Descritores: Sistemas de saúde; Saúde da família; Educação superior; Formação de recursos humanos; Enfermagem; Medicina; Odontologia

\begin{abstract}
The establishment and implementation of the Brazilian universal healthcare system (Sistema Único de Saúde [SUS]) guarantees universality, fairness, and establish healthcare as a duty of the state. Several strategies have been developed, including the Family Health Program which in theory redefines primary care. However, there are weaknesses in the professional education of nurses, physicians, and dentists to provide primary care to the individual, family, and community as defined by the SUS. Therefore, humanization of care becomes a paradox. The authors discuss those issues using narrative review and emphasizes Freire's theoretical framework as an approach for college education of healthcare professionals to overcome difficulties in adopting and implementing primary care according to ideals and principles of the SUS. Keywords: Health systems; Family health; Education, higher; Human resources formation; Nursing; Medicine; Dentistry
\end{abstract}

\section{RESUMEN}

La creación e implementación del Sistema Único de Salud (SUS) trajo victorias como la universalidad, equidad e integralidad en la asistencia como un deber del Estado. Diversas estrategias fueron concebidas, inclusive el Programa de Salud de la Familia que en teoría re-significaría la atención primaria en el ámbito del SUS. Entre tanto, se constata deficiencias en la formación universitaria del enfermero, del médico y del odontólogo por la dificultad de estos profesionales en la reorientación de las acciones que enfoquen a la familia y la promoción de la salud, dentro de la necesidad de actuación integral. En este interin, la temática de la humanización de la atención se vuelve en un emblema privilegiado de la paradoja. Los autores debaten estas cuestiones a través del uso de la revisión narrativa, promoviendo la reflexión sobre el marco teórico freireano como camino en la formación universitaria para la superación de estos dilemas en la consecución del SUS de acuerdo a sus ideales y principios

Descriptores: Sistemas de salud; Salud de la familia; Educación superior; Formación de recursos humanos; Enfermería; Medicina; Odontologia

\footnotetext{
1 Doutor, Professor Adjunto do Departamento de Saúde Pública da Universidade Federal de Santa Catarina - UFSC - Florianópolis (SC), Brasil.

${ }^{2}$ Doutora, Livre-Docente do Departamento de Enfermagem Psiquiátrica e Ciências Humanas da Escola de Enfermagem de Ribeirão Preto da Universidade de São Paulo - USP - Ribeirão Preto (SP), Brasil.
} 


\section{INTRODUÇÃO}

Na história do Brasil destacam-se três momentos com relação à garantia de acesso à saúde pelo Estado: o início do século XX: as ações estatais em saúde se referiam apenas a campanhas contra epidemias em regiões geográficas de interesse comercial, tais como portos e capitais; o segundo momento: o Estado garantia ações para trabalhadores dos setores de interesse econômico, mediante o pecúlio obrigatório, que, posteriormente, se estendeu a todos os trabalhadores - modelo preservado até o colapso do Sistema Nacional de Saúde na década de $1980^{(1)}$; momento atual: inicia-se em 1988 a partir da mudança da relação entre Estado/cidadãos, com a inserção de princípios na Constituição Nacional como o direito à saúde a todos os cidadãos sendo dever do Estado, através do Sistema Único de Saúde (SUS) ${ }^{(2)}$.

A universalização do acesso aos serviços promove avanços em termos de Saúde Coletiva. No entanto, em sensu stricto, os princípios doutrinários do SUS foram corrompidos na construção do Sistema de fato, que não atingiu um estágio de maturidade em que se tornam visíveis os princípios norteadores e filosóficos do SUS.

Em função deste contexto, amplo debate entre Governo, intelectuais e sociedade foi promovido durante a década de 1990, ocasionando reorganização do processo de produção dos serviços de saúde de maneira profunda e da estruturação do sistema em si.

Este processo, que primou pelo refinamento do SUS existente em consonância com as diretrizes e princípios do SUS desejado ${ }^{(3)}$, culminou na criação do Programa de Saúde da Família, estratégia rumo à idealização e valorização da prestação de serviços públicos de saúde articulada junto a própria comunidade, não apenas enfocando ações curativas e centradas na doença, mas, em especial, pautado em ações que incidissem sobre determinantes de saúde e promoção de saúde ${ }^{(4)}$. Nesta nova abordagem da atenção primária no Brasil, prima-se pelo paciente como parte importante para estabelecimento não apenas do diagnóstico clínico, mas também para o entendimento do processo saúde-doença e para intervenção junto aos múltiplos determinantes, re-significando e valorizando o papel do usuário junto ao Sistema ${ }^{(4)}$.

Neste contexto de transformação, o papel dos profissionais de saúde também foi re-significado para a atenção primária ${ }^{(4)}$. Mais do que prestador de serviços junto a ações para a cura e a reabilitação, a nova política de saúde exige um profissional adequado à consecução dos princípios do SUS na prática diária. E essa exigência não se refere apenas às novas categorias profissionais como os agentes comunitários de saúde. Refere-se, particularmente, às categorias tradicionais, como a Enfermagem, assim como aquelas de cunho liberal, como a Medicina e a Odontologia.
Com ampla literatura para cada corporação mencionada, a questão da formação dos profissionais e sua adequação para o SUS ganhou força devido a formação universitária primar pela prática individual e centrada no hospital como locus de atuação privilegiada para o enfermeiro e para o médico, assim como no consultório particular para o odontólogo; formação divergente daquela necessária à implementação do SUS de fato, não apenas em teoria.

Não obstante a existência de diversos artigos, dissertações e teses sobre a referida temática para cada uma das três profissões, e as freqüentes denúncias sobre a desarticulação diante das necessidades do SUS para as referidas profissões, a literatura é exígua em trabalhos que analisem as três profissões em conjunto no âmbito da formação para o SUS, assim como mostra lacuna no sentido de caminhos possíveis que solucionem a problemática.

Em vista ao exposto, a proposta do presente artigo foi a de apresentar a literatura sobre a desarticulação entre a formação do enfermeiro, do médico e do odontólogo para o SUS e a pertinência do marco teórico de Paulo Freire como modelo pedagógico universitário em saúde, para a superação desta desarticulação.

\section{MÉTODOS}

Para tanto, utilizou-se do método de revisão narrativa, que se dispõe principalmente a levantar, com caráter qualitativo, fundamentação existente na literatura científica em questões consideradas amplas ou abertas. Dada a amplitude, a revisão narrativa não possui um protocolo padrão para sua elaboração. Em termos metodológicos, busca informações atualizadas sobre determinada temática de trabalhos científicos de outros autores, sedimentando fundamentação teórica para determinada questão, de forma a descrever e discutir o conhecimento existente sobre assunto a que se propõe, contextualizando o leitor sobre o tema. Constitui-se, portanto, em análise da literatura vigente, a partir do pensamento crítico dos autores da revisão.

No presente estudo, foram selecionados artigos, documentos oficiais, dissertações, teses do campo da Saúde Coletiva que se referiam à formação do enfermeiro, do médico e do odontólogo para o SUS, assim como aqueles que se inseriam neste debate. Também utilizaram-se os princípais trabalhos de Paulo Freire para levantar o universo conceitual deste teórico.

\section{DESENVOLVIMENTO}

A partir das discussões estabelecidas entre os diversos atores em Saúde Coletiva na década de 1990, como já referido anteriormente, legitimou-se a criação, implementação e expansão do Programa de Saúde da Família como modelo de atenção primária no Brasil, 
tendo em vista a superação do modelo de atenção anterior, incompatível com os princípios do SUS.

O processo propiciou significativa reorganização dos trabalhadores de saúde em Unidades de Saúde da Família, pretendendo reestruturar a atenção primária na direção contrária à fragmentação e desarticulação entre os profissionais no modelo de atenção tradicional, preconizando o trabalho em equipe multiprofissional que, em teoria, propiciaria atenção em consonância com os princípios do SUS. Neste novo enfoque, preconiza-se tratamento horizontalizado entre equipe e usuário, com postura de similaridade, proximidade e acolhimento das demandas biológicas, emocionais e humanas dos pacientes ${ }^{(5)}$.

A centralidade da família nas ações do Programa de Saúde da Família (PSF) reorienta a atenção básica não apenas em termos da organização das unidades de saúde, mas principalmente na adoção de novo paradigma para o processo saúde-doença, em que determinantes de saúde não estritamente biológico-comportamentais emergem na discussão sobre a intervenção dos profissionais de saúde.

Em termos de equidade e universalidade, o PSF mostrouse como avanço rumo a efetividade do SUS como preconizado, dada a implantação do programa em regiões com problemas de acesso geográfico aos serviços de saúde ${ }^{(6)}$.

No entanto, com relação ao princípio da integralidade, a estratégia não teve os mesmos resultados, uma vez que este princípio entra em questões como a humanização da atenção, vínculo entre profissionais e usuários, coresponsabilização deste atores na resolução dos problemas de saúde, além da imersão do profissional na comunidade ${ }^{(7)}$.

As ações integrais e de enfoque para a promoção da saúde, tais como as visitas domiciliares e outras atividades que permitam vivenciar a realidade em que intervirá, são preconizadas para todos os profissionais ${ }^{(8)}$, focando a atuação na equipe multiprofissional, no paciente enquanto ser que vive e é articulado em múltiplas interfaces sociais, econômicas e culturais, e que deve ter participação no planejamento das ações junto com a equipe. Na prática, as equipes de Saúde da Família, mesmo com composição multiprofissional, não conseguirão implementar as ações tal como ensejada para o $\operatorname{PSF}^{(9)}$.

Em grande medida, as falhas neste processo de implementação referem-se à formação do recurso humano em saúde, dado que o profissional em seu dia-adia apresenta dificuldades para operacionalizar os conceitos de integralidade e do enfoque atual em Saúde Coletiva como ação nos serviços ${ }^{(10)}$.

Há indícios de que as iniciativas da equipe para manter postura humanizada, conforme preconizada em documentos oficiais da estratégia, são superficiais e até mesmo conflituosas ${ }^{(11)}$, havendo dificuldades para o profissional valorizar as inter-relações humanas, permanecendo em uma abordagem biomédica na prática no serviço público, distando do talhe necessário para a atuação nas Unidades de Saúde da Família ${ }^{(12)}$.

O contexto que se mostra é que a transformação legal da atenção primária no país, direcionada à integralidade e a humanização, não garante proximidade entre a teorização do PSF e o processo de trabalho de fato nos serviços de saúde $^{(11)}$. Paralelamente ao paradoxo nos serviços, as transformações não são acompanhadas pela formação dos profissionais de saúde da equipe mínima nas Instituições de ensino superior (IES), segundo a literatura ${ }^{(10-}$ 14), tanto nos aspectos da ação clínica como nos que se referem a criar habilidade para vínculo e humanização do atendimento, questão implicada na formação do profissional também como cidadão ${ }^{(15-17)}$.

A importância desta temática é tamanha que ganha importância no cenário de políticas públicas de saúde do Brasil, não se restringindo ao contexto do $\mathrm{PSF}^{(18-19)}$. Em 2003, o governo federal instituiu a Política Nacional de Humanização do SUS - HumanizaSUS ${ }^{(20)}$ e em 2005 o Programa Nacional de Reorientação da Formação Profissional em Saúde - Pró-Saúde ${ }^{(21)}$, que aponta para a necessidade de reformulação principalmente na enfermagem, medicina e odontologia para sua inserção na equipe mínima do PSF, sendo indissociável a questão da formação, para que, efetivamente, o SUS venha traduzir o que se preconiza teoricamente ${ }^{(22)}$.

No seio da universidade, a concepção pedagógica que vigora nos cursos de graduação e que alicerçará os futuros enfermeiros, médicos e odontólogos, mantém-se centrada na visão reducionista da saúde e da doença. Apesar de contraditória ao novo enfoque para os profissionais de saúde, ainda é a perspectiva predominante entre docentes, formadores destes futuros profissionais ${ }^{(23)}$.

A formação universitária ainda mantém-se distante do enfoque totalizador de ser humano, permanecendo centrada no modelo de ensino das técnicas e no desenvolvimento restrito de competências, sem legar, ao futuro profissional, raciocínio crítico reflexivo para ação junto ao paciente e no contexto social, modulador da e modulado pela sociedade em que e com a qual está vivendo ${ }^{(24)}$.

A desarticulação entre a formação universitária e a necessidade do SUS denuncia a premência de formação para a atenção integral e holística durante a construção profissional em saúde, articulada com a realidade, o que é amplamente defendido na literatura científica ${ }^{(24-25)}$.

O perfil do enfermeiro, do médico e do odontólogo generalista e humanista, paralelamente a atuação técnicocientífica, não é consonante com o perfil dos egressos das IES, apesar das ações do Estado, assim como das próprias IES. Na prática cotidiana do PSF, prevalece o despreparo dessas três categorias profissionais para o modelo de Saúde da Família ${ }^{(11,26)}$.

No ensino de enfermagem destacam-se, entre os nós críticos, a fragmentação entre a teoria e a prática de enfermagem, desarticuladas também das questões do 
novo contexto de saúde no Brasil ${ }^{(27)}$; dificuldade de superação do modelo tradicional em vista das atuais necessidades em saúde ${ }^{(28)}$; falta de reorientação da formação para uma visão crítica, pautando-se apenas na técnica ${ }^{(29)}$; necessidade de reflexão sobre os papéis, tanto do docente como do discente no processo ${ }^{(30)}$.

Sobre o ensino médico, as dificuldades referem-se à articulação entre conhecimento técnico-biológico e ação interdisciplinar em direção à complexidade das necessidades dos pacientes em saúde ${ }^{(31)}$; garantia que o ensino de graduação efetivamente construa alicerce sólido para a capacitação posterior à graduação ${ }^{(32)}$; superação do hospital como centro privilegiado na formação do médico, mudando o enfoque para a atenção primária ${ }^{(33)}$; necessidade de adoção de medidas integradoras entre academia e assistência, desde o início da formação profissional dos estudantes de medicina ${ }^{(34)}$; busca por estratégias norteadoras para a interdisciplinaridade e o trabalho em equipe, além da atenção humanizada ${ }^{(35)}$.

A formação do odontólogo apresenta como desafios a serem superados: caminhos para pautar modelo pedagógico em uma formação humanística e ética, não apenas na execução clínica, assim como na promoção de saúde pautada em prática odontológica interdisciplinar no âmbito coletivo ${ }^{(36)}$; distanciamento do caráter essencialmente tecnicista da formação ${ }^{(37)}$; articulação entre educação superior e serviços de atenção a saúde e nos princípios do SUS ${ }^{(38)}$; re-adequação do perfil do odontólogo egresso às necessidades e demandas populacionais e não apenas dos que podem recorrer ao serviço em consultório particular ${ }^{(39)}$.

Apesar de ampla literatura que aponte a premente necessidade de formar profissionais de saúde em outro enfoque, há ausência de discussão sobre como seria este modelo em termos teóricos e operacionais ${ }^{(37)}$. Há lacunas sobre como formar o enfermeiro, o médico e o odontólogo de forma a possibilitar a integração das profissões numa genuína equipe multiprofissional em Saúde Coletiva, com maior coerência entre o que o SUS é e o que deveria ser, por seus princípios ${ }^{(39)}$.

Tendo em vista que a formação do enfermeiro, do médico e do odontólogo no âmbito de sua atuação no PSF apresenta lacunas quanto a abordagem holística do paciente, de seu entorno sócio-cultural e dos determinantes do processo saúde-doença, o marco teórico de Paulo Freire sobre humanização torna-se pertinente.

Cabe ressaltar, que no marco teórico freireano, humanizar não significa assistencialismo, ou apenas simpatia, ou signifique que o profissional ignore as contradições e os problemas existentes no seu campo de trabalho, apenas tratando o paciente de forma agradável ${ }^{(16-17)}$. Diametralmente a esta postura alienada, no universo conceitual freireano o ser humano humanizado é cidadão, seja profissional de saúde ou usuário do sistema, que se vê com poder de atuar em sociedade pelo processo de conscientização que, justamente, demanda refletir sobre contradições e problemas do cotidiano.

A postura humanizada freireana reflete o entendimento de que, antes de ser um profissional, é um ser humano e como tal, não escapa da relação oprimido/opressor na qual estão inseridos todos os homens que vivem no modo de produção capitalista. Homens oprimidos por não poderem ser humanos em sentido amplo ${ }^{(16,17)}$.

Há que se registrar que são características peculiares ao marco teórico freireano: o diálogo entre homens que estão no mundo, moduladores e modulados por este mesmo mundo e pelas relações com seus semelhantes; o significado do ensino e da aprendizagem como ato político e modificador do mundo; a educação como visão crítica social da realidade e dos processos humanos; a centralidade do tema humanização ${ }^{(16-17,39)}$.

Como apresentado anteriormente, na efetivação do PSF e do SUS há necessidade de modelo em que o profissional atue para a integralidade, modelo de humanização, consoante com abordagem crítica social e reflexiva, humanizada e dialógica. Trata-se de necessidade, portanto, de pensamentos significativamente pertinentes ao pensamento freireano, uma vez que a temática da relação horizontal/vertical entre paciente e profissional não é distante de outra: a relação entre professor e aluno na graduação e suas conseqüências na postura profissional( ${ }^{(24)}$.

Conforme o universo conceitual freireano, não se aprende democracia, se não se exerce democracia. Não se aprende cidadania, se não se exerce cidadania ${ }^{(16-17)}$. E não se aprenderá o exercício profissional que prime pelos princípios do SUS e pelo processo de trabalho intersetorial e multiprofissional se, no âmbito privilegiado da formação universitária, o futuro enfermeiro, o futuro médico e o futuro odontólogo não exercerem cada etapa da formação universitária de forma consonante com estes princípios e junto às demais formações ${ }^{(39)}$.

Nesta visão sobre estas profissões e seu processo de trabalho em consonância com o SUS no Programa em Saúde da Família, a peculiaridade do processo de trabalho multiprofissional, precisa de um olhar amplo. Não apenas individualizado na corporação profissional, mas sim focada na formação de equipe de profissionais, respeitadas as diversas singularidades pertinentes às diversas áreas, mas com um enfoque único, na ação junto à sociedade, às famílias e na promoção da saúde dos indivíduos. Não simplesmente na cura e na prestação do serviço técnico ${ }^{(39)}$, mas sim no refletir sobre os serviços de saúde, que não se distancia da abordagem crítica social e reflexiva, humanizada e dialógica dos pressupostos freireanos ${ }^{(16-17)}$.

\section{CONSIDERAÇÕES FINAIS}

Há lacunas na literatura quanto a operacionalização 
para formar o enfermeiro, o médico e o odontólogo satisfatoriamente em relação aos princípios norteadores do SUS, em que se pese a necessidade de interdependência do ensino e da assistência na formação para a integralidade, trazendo como referência básica e fundamental o cuidado do usuário do sistema. Apesar da ausência de caminhos para tal, prima-se pela construção de uma formação diferenciada da tradicional, integrando a equipe enfermeiro, médico, odontólogo e outros profissionais e o paciente no contexto do SUS.

Dadas as necessidades, principalmente no que concerne a formação holística e humanizadora dos profissionais de saúde, a perspectiva dialógica do marco teórico freireano se mostra um caminho possível.

Os pressupostos de Paulo Freire e do marco teórico que estabeleceu podem se constituir em direcionamento para a reorganização da formação de saúde com o enfoque humano, não distanciando o contexto da formação

\section{REFERENCIAS}

1. Elias PE. Estado e saúde: os desafios do Brasil contemporâneo. São Paulo Perspect. 2004;18(3):41-6.

2. Silva PLB. Serviços de Saúde: o dilema do SUS na nova década. São Paulo Perspect. 2003;17(1):69-85.

3. Vasconcelos CM. Paradoxos da mudança no SUS [tese]. Campinas (SP): Faculdade de Ciências Médicas da Universidade Estadual de Campinas; 2005.

4. Aciole GG. Das dimensões pedagógicas para a construção da cidadaniano exercício do controle social. Interface Comum Saúde Educ. 2007;11(23):409-26.

5. Brasil. Ministério da Saúde. Secretaria de Gestão do Trabalho e da Educação na Saúde. Departamento de Gestão da Educação na Saúde. Política de educação e desenvolvimento para o SUS: caminhos para a educação permanente em saúde. Brasília: Ministério da Saúde; 2004.

6. Alves VS. Um modelo de educação em saúde para o Programa Saúde da Família: pela integralidade da atenção e reorientação do modelo assistencial. Interface Comum Saúde Educ. 20042005;9(16):39-52.

7. Schimith MD, Lima MADS. Acolhimento e vínculo em uma equipe do Programa Saúde da Família. Cad Saúde Pública = Rep Public Health. 2004;20(6):1487-94.

8. Brasil. Ministério da Saúde. Secretaria de Políticas de Saúde. Departamento de Atenção Básica. A implantação da Unidade de Saúde da Família. Brasília:Ministério da Saúde; 2000.

9. Ramos DD, Lima MADS. Acesso e acolhimento aos usuários em uma unidade de saúde de Porto Alegre, Rio Grande do Sul, Brasil. Cad Saúde Pública = Rep Public Health. 2003;19(1):27-34.

10. Pinheiro R. Práticas de saúde e integralidade: as experiências inovadoras na incorporação e desenvolvimento de novas tecnologias assistenciais de atenção aos usuários no SUS. In: Brasil. Ministério da Saúde. Secretaria de Gestão de Investimentos em Saúde. Projeto Reforsus - Componente II. Experiências Inovadoras no SUS: relatos de experiências: novas tecnologias assistenciais: Secretarias Estaduais e Municipais de Saúde. Brasília: Ministério da Saúde; 2002. p.15.

11. Puccini PT, Cecílio LCO. A humanização dos serviços e o direito à saúde. Cad Saúde Pública $=$ Rep Public Health. 2004;20(5):1342-53. profissional da necessidade de um tom de formar tendo em vista as atuais perspectivas da prática de saúde nesta reorganização de práticas. De formar para o SUS.

Uma vez que o presente trabalho trata-se de uma revisão narrativa, é importante se ressaltar no âmbito das considerações finais que são necessários estudos no campo da formação do profissional de saúde, em particular do enfermeiro, do médico e do odontólogo. Não simplesmente indicando as deficiências, haja vista o amplo número de trabalhos que o fazem em cada uma das profissões estudadas aqui, mas sim o estabelecimento de estudos que realizem análise das três profissões conjuntamente, uma vez que é assim que se articularão no cerne do processo de trabalho da Saúde da Família, tanto quanto que se pesquisem caminhos e modelos pedagógicos que possibilitem formar para o SUS, sendo que o marco teórico freireano pode se constituir como tal.

12. Tesser CD. Medicalização social (II): limites biomédicos e propostas para a clínica na atenção básica. Interface Comum Saúde Educ. 2006;10(20):347-62.

13. Gomes MCPA, Pinheiro R. Acolhimento e vínculo: práticas de integralidade na gestão do cuidado em saúde em grandes centros urbanos. Interface Comum Saúde Educ. 2005;9(17):287-302.

14. Merhy EE. Em busca do tempo perdido: a micropolítica do trabalho vivo em saúde. In: Merhy EE, Onocko R, organizadores. Agir em saúde: um desafio para o público. São Paulo: Hucitec; 1997. p. 71-112.

15. Markman Neto L. Política de saúde, sistema único de saúde e a prática do programa de saúde da família num núcleo específico. (Dissertação). Ribeirão Preto: Faculdade de Medicina de Ribeirão Preto da Universidade de São Paulo; 2004.188p.

16. Freire P. Pedagogia do oprimido. 43a ed. São Paulo: Paz e Terra; 1999.

17. Freire P. Pedagogia da autonomia: saberes necessários à prática educativa. São Paulo: Paz e Terra; 1999.

18. Simões ALA, Rodrigues FR, Tavares DMS, Rodrigues LR. Humanização na saúde: enfoque na atenção primária. Texto \& Contexto Enferm. 2007;16(3):439-44.

19. Brasil. Ministério da Saúde. Secretaria de Assistência à Saúde. Programa Nacional de Humanização da Assistência Hospitalar. Brasília: Ministério da Saúde; 2001.

20. Brasil. Ministério da Saúde. Secretaria de Atenção à Saúde. Departamento de Atenção Básica.Coordenação Nacional de Saúde Bucal. Projeto SB Brasil 2003: condições de saúde bucal da população brasileira 2002-2003: resultados principais. Brasília: Ministério da Saúde; 2004.

21. Brasil. Ministério da Saúde. Ministério da Educação. Programa Nacional de Reorientação da Formação Profissional em Saúde: Pró-Saúde: objetivos, implementação e desenvolvimento potencial. Brasília: Ministério da Saúde; 2005.

22. Bosi MLM. Cidadania, participação popular e saúde na visão dos profissionais do setor: um estudo de caso na rede pública de serviços. Cad Saúde Pública = Rep Public Health. 1994;10(4):446-56.

23. Vaitsman J. Saúde, cultura e necessidades. In: Fleury S, organizador. Saúde: Coletiva? Questionando a onipotência 
do Social. Rio de Janeiro: Relume Dumará; 1992. p.157-73.

24. Caprara A, Franco ALS. A relação paciente-médico: para uma humanização da prática médica. Cad Saúde Pública $=$ Rep Public Health. 1999;15(3):647-54.

25. Pierantoni CR, Machado MM. Profesiones de salud: una formación cuestionada. Educ Méd Salud. 1994;28(2):199210 .

26. Brasil. Ministério da Saúde. Ministério da Educação. A aderência dos cursos de graduação em enfermagem, medicina e odontologia às diretrizes curriculares nacionais. Brasília: Ministério da Saúde: Ministério da Educação; 2006.

27. Scherer ZAP, Scherer EA. Reflections on nursing teaching in the post-modernity era and the metaphor of a theory-practice gap. Rev Latinoam Enferm. 2007;15(3):498-501.

28. Pinto JBT, Pepe AM. Nursing education: contradictions and challenges of pedagogical practice. Rev Latinoam Enferm. 2007;15(1):120-6.

29. Freitas DMV, Fávero N, Scatena MCM. O ensino de graduação na Escola de Enfermagem de Ribeirão Preto da Universidade de São Paulo: suas prospectivas. Rev Latinoam Enferm. 1993;1(N Esp):25-34.

30. Silva AL, Camillo SO. A educação em enfermagem à luz do paradigma da complexidade. Rev Esc Enferm USP. 2007;41(3):403-10.

31. Koifman L. O modelo biomédico e a reformulação do currículo médico da Universidade Federal Fluminense. Hist Ciênc Saúde - Manguinhos. 2001;8(1):48-70.

32. Coêlho BM, Zanetti MV, Lotufo Neto F. Residência em psiquiatria no Brasil: análise crítica. Rev Psiquiatr Rio Gd
Sul. 2005;27(1):13-22.

33. Blank D. A propósito de cenários e atores: de que peça estamos falando? Uma luz diferente sobre o cenário da prática dos médicos em formação. Rev Bras Educ Méd. 2006;30(1): 27-31.

34. Noronha Filho G, Resende JB, Lemme AC, Ney Júnior G, Frossard A. Formação médica e integração de atividades docentes e assistenciais. Rev Saúde Pública $=\mathrm{J}$ Public Health 1995;29(3):215-20

35. Pontes AL, Rego S, Silva Júnior AG. Saber e prática docente na transformação do ensino médico. Rev Bras Educ Med. 2006;30(2):66-75.

36. Nuto SAS, Noro LRA, Cavalsina PG, Costa ICC, Oliveira AGRC. O processo ensino-aprendizagem e suas conseqüências na relação professor-aluno-paciente. Ciênc Saúde Coletiva. 2006;11(1):89-96.

37. Freitas SFT, Kovaleski DF, Boing AF. Desenvolvimento moral em formandos de um curso de odontologia: uma avaliação construtivista. Ciênc Saúde Coletiva. 2005;10(2):453-62.

38. Matos PES, Tomita NE. A inserção da saúde bucal no Programa Saúde da Família: da universidade aos pólos de capacitação. Cad Saúde Pública = Rep Public Health. 2004;20(6):1538-44.

39. Pires ROM. O pensamento crítico social de Paulo Freire sobre humanização e o contexto da formação do enfermeiro, do médico e do odontólogo [tese]. São Paulo: Escola de Enfermagem de Ribeirão Preto da Universidade de São Paulo; 2008. 